\title{
An Interview with Ronald M. Evans, Ph.D. Investigator, The Salk Institute for Biological Studies
}

\begin{abstract}
Dr. Ronald M. Evans is known for his original discoveries of nuclear receptor hormone receptors and the elucidation of their universal mechanism of action, a process that governs how lipophilic hormones and drugs regulate virtually every developmental and metabolic pathway in animals and humans. Dr. Evans obtained his Ph.D. in microbiology and immunology from the University of California, Los Angeles, School of Medicine and was a postdoctoral fellow at the Rockefeller University in New York. At Rockefeller, his studies on the transcriptional regulation of human adenovirus led to the identification of the first eukaryotic promoter for messenger RNA. In 1977, he joined the faculty of The Salk Institute for Biological Studies where he is an Investigator of the Howard Hughes Medical Institute and Professor in the Gene Expression Laboratory. He also holds the March of Dimes Chair in Molecular and Developmental Biology. In addition to his Salk appointment, he is an Adjunct Professor at the University of California, San Diego in the Departments of Biology, Biomedical Sciences, and Neuroscience. He was elected to the National Academy of Sciences in 1989, was named the 1994 California Scientist of the Year by the California Museum

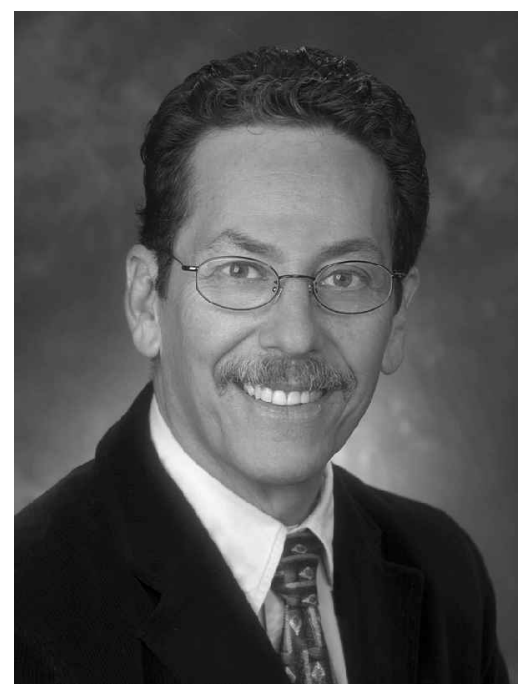
of Science and Industry, and was elected to the American Academy of Arts and Sciences in 1997 and the Institute of Medicine of the National Academies in 2003. Consistent with the broad impact of his work, he is listed by the Institute of Scientific Information as one of the most cited scientists of the past decade.
\end{abstract}

\section{Dr. Evans, how large is the nuclear receptor super- family?}

There are 48 genes in the human genome that comprise the nuclear receptor superfamily (not including splice variants); perhaps more important than the number of genes is the discovery that a superfamily even exists, which is one of the true achievements of this field. At first, all we knew was that steroid receptors existed. The discovery that they were part of a superfamily was very powerful, because it suggested that there were more receptors than we originally thought, and that all of the members of the superfamily had a common structure (based on their homology) and, therefore, they must act through a common mechanism. This told us that the other members of the superfamily worked in the same way as the steroid receptors.

This discovery of a superfamily, which was one of the unexpected parts of the adventure, led to a number of questions: why should there be a family, why does it con- tain this particular number of genes, and do more hormones exist? There certainly does appear to be a grand design, and as far as we know, all nuclear hormonal lipids act through this superfamily. This is conceptually different from cell surface receptors, which employ second messenger pathways, such as tyrosine kinases, GPCRs (G protein-coupled receptors), cAMP signaling, and other types of signal transduction mechanisms. For the nuclear receptors, there is only one pathway.

What are some of the drugs and their indications that have been developed to target these receptors, and do you expect nuclear receptors to grow significantly as a class of drug targets for the pharmaceutical industry?

That is a very broad-ranging question. The most commonly used drugs are the contraceptives, as well as estrogens for hormone replacement therapy, antiestrogens to treat breast and ovarian cancer, androgens, anabolic steroids, and antiandrogens for prostate cancer. Next are 
the corticoids, which are used to treat inflammation, arthritis, and asthma, eplerenone for hypertension, and RU-486 (the morning-after pill). Thyroid hormone (e.g., synthroid) is widely prescribed, and vitamins A and D are used in many fortified foods. Thus, it is virtually impossible for people not to take ligands for nuclear receptors on a daily basis. They are available as treatments of disease and also for their health and lifestyle effects. In addition, many antidiabetic drugs and lipid-lowering drugs also target nuclear receptors. The total market for drugs that target nuclear receptors is about $\$ 12$ million, and that market will continue to grow.

Selective estrogen modulators (SERMS) now have an important place in the pharmacy. An optimistic view of nuclear receptors as drug targets would hold that selective modulators for other nuclear receptors might yield drugs that have the benefits of known hormones without the side effects. Do you see a rational way forward to discovering and developing selective nuclear receptor modulators, and if so, what is that path?

The SERMS represent synthetic molecules that modulate the functional activity of the estrogen receptor in a fashion that is distinct from the natural hormone. SERMS do have an important place in the pharmaceutical industry and have led to a new view of how nuclear receptors may be targeted for the development of drugs. The SERMS are conceptual prototypes for other receptors. The SERMS themselves are used in women's health to treat osteoporosis and breast and ovarian cancer. Tamoxifen is the prototypic SERM, but new generations of drugs are already coming through. Raloxifene is the first of the new generation of SERMS, which are more selectively targeted to reduce side effects and are active in a restricted number of tissues. Designing a hormone that instead of acting in every tissue acts in only selected tissues is one of the goals of SERM development. If you can antagonize estrogen in the breast, but not antagonize its action in the bone, then you can block breast cancer but not cause bone degeneration. The SERMS act as agonists in one tissue and antagonists in another.

That ability probably extends to any other nuclear receptor as well. The effort to develop modulators for the glucocorticoid receptor is under way, as are programs targeting the aldosterone receptor for hypertension and PPARs (peroxisome proliferator-activated receptors) for antidiabetic drugs. As a result, in the next few years we will probably see at least a half dozen new estrogen drugs with targeted applications that are safer for bone, promote better heart effects, and are more effective in preventing or treating breast cancer. Estrogen is part of every woman's life, and the majority of women will take some form of estrogen, whether in their early years as contra- ceptives or in their later years as hormone replacement, or some form of estrogenic therapy for relatively common diseases such as breast cancer.

As a group, the nuclear receptors represent one of the most widely prescribed classes of drugs. For the treatment of diabetes, the PPAR agonists, called TZDs (thioazolidinediones) or glitazones, such as rosiglitazone (Avandia) and pioglitazone (Actos), alone comprise a market of about $\$ 4$ billion. They are first generation drugs. The second generation forms of those drugs, with reduced side effects, called SPARMS (selective PPAR modulators), are already in advanced development. TZDs cause water retention (edema) and weight gain. Those properties are being designed out of the newer drugs, and their insulin sensitization capability is being improved.

What are the implications of using reporter gene cell lines versus ligand binding assays in screening for modulators of nuclear receptor activity?

The question really is how can you use nuclear receptors to find new drugs? Once the receptors were cloned and their target genes identified, a strategy was immediately devised that incorporated a very high throughput screening system. These cell-based screens, aimed at identifying agonists and antagonists of the target, have been very effective for identifying new drugs and evolving those drugs for improved specificity and sensitivity. The screening process is fast, cheap, and precise. The disadvantage is that the screens do not tell you a lot about the physiology of the drugs in the animal. For that, you have to put the drug into the animal.

Reporter assays have transformed the industry. You cannot reasonably screen a library of compounds in animals. You need a high throughput screening method. In vitro assays can also be very powerful; however, cellbased reporter assays have several advantages: they have very high sensitivity, they screen for molecules that can get into cells that are not toxic, and they can tell you if you have an agonist or an antagonist.

With cell-based assays, are there any disadvantages or advantages in using endogenous versus transfected (overexpressed) nuclear receptors?

The transfected approach is probably the easiest, because it allows comparisons of many different receptors in the same cell line within the context of the same experiment. If you have a stable endogenous cell line with a stable reporter, it will be effective for an individual receptor. However, for comparison purposes, you would need to develop such a cell line for each receptor. The default is to work with a transient transfection. It is extremely fast, highly quantitative, and does not require creating cell lines. 
One of the main challenges in targeting nuclear receptors is finding/characterizing nuclear receptor modulators. It is believed that those modulators could induce different conformational states that could in turn recruit different coactivators, resulting in differential biological effects. What is the best assay to probe different conformations? Can the difference in coactivator tissue distribution lead to identification of tissue-selective nuclear receptor modulators?

These receptors are transcriptional modulators. Transcription is modulated not so much by the transcription factor itself, but by the coactivators or corepressors they attract. The receptors are like magnets that recruit coactivators or corepressors in response to a ligand. Engineered ligands alter the conformational properties of the receptors in ways that are different from natural molecules. As a result, you can exploit that to build new properties into these synthetic compounds. Each ligand is unique; no two chemicals bind exactly alike, and they will not bind the natural hormone in exactly the same way.

This opens up a whole new world. Cofactors change in different tissues. While the receptor itself may be common across tissue types, the context of the coactivators or regulators may be tissue-specific. Therefore, different types of ligands can acquire tissue-specific properties that are different from the endogenous hormone. Pharmacology can take advantage of the cofactors to create a diversity of compounds. This is driving the future of the field and presents a large opportunity. However, although you may be able to generate new compounds, you may not discover a useful compound quickly. That is why drug development has become linked to molecular biology. Molecular biologists study receptor structure, receptor conformation, and cofactor signaling. Pharmaceutical companies are becoming increasingly interested in traditional molecular dissection of transcription. This is where you can apply the sophisticated combination of pharmacology, chemistry, and molecular biology to discover new generations of compounds.

The best example of this ability to undergo conformational changes is the estrogen receptor, because compounds targeting the receptor already exist, such as tamoxifen and raloxifene. Crystal structures of the receptors bound to those ligands reveal the different conformational structures. This explains why the two compounds act differently and demonstrates that the ligand causes the conformational change. About 100 coactivators have been identified, and they are promiscuous for all of the members of the nuclear receptor superfamily.

\section{What are the best assays for probing different receptor conformations?}

A number of assays have been designed to look at conformational changes. These involve selectively test- ing the recruitment of individual coactivators one at a time and quantifying them relative to the natural ligand. Another approach is to use reporter genes and look for compounds that function as partial agonists or partial antagonists. They would demonstrate high-affinity binding, but would not activate the receptor to the $100 \%$ level. Partial activation occurs because the ligand is not recruiting the full complement of cofactors. Finally, people have specifically designed peptides that decorate the surface of a receptor. The ability of compounds to attract or repel these peptides creates a fingerprint that is unique to that compound. This is an effective screening method.

\section{Please describe the events that led your laboratory in 1985 to clone the first steroid hormone receptor.}

It is impossible to briefly describe those events, but I will try to give you a broad view of what was happening at that time. It was one of the most exciting times in my career. It was the core project I chose to work on after I left my postdoc with Jim Darnell at Rockefeller in New York. I focused my efforts on glucocorticoid and thyroid hormones, and one of my long-term goals was to isolate the receptors that we believed would act as a type of genetic switch, controlled by those two classes of hormones.

At the time that we did this work, no transcription factor had been cloned. Soon after, however, many would be cloned, but at that stage it was a wild ride. Not the least part of the adventure was the competition. A lot of outstanding laboratories were pursuing this problem. The glucocorticoid and estrogen receptors were the two prototypes. We used a complicated set of phage expression libraries-libraries that produced protein fragments-and we repeatedly screened them with antibodies. Based on the findings, we created new libraries. I had two outstanding people in my lab, and we were basically living in the lab at that time, working day and night.

We laid out a strategy, and despite my enthusiasm it unfortunately seemed like it was not working. I had a stressed postdoc and a very focused graduate student. Finally, we said, "Let's give it one more shot," and we screened another million clones. One film showed a tiny dot on it, about the size of a millimeter, and I remember Cary Weinberger, the postdoc who did the work, say, "This is it!" In fact, he was right. We then began the complex process of sequencing that clone.

At the time, rumors were swirling around that one of our competitors had found something, and I'm sure there were rumors going around that we had found something as well. The pressure was intense. Luckily for us, we had isolated virtually a full-length clone, which is the most difficult form to get. I left my two associates in the lab to sequence the clone and went off to the Gordon Conference back East. They managed to complete the se- 
quence while I was gone. In 1985, we were working with a colleague named Russ Doolittle, who had created one of the first sequence databases-you had to $\log$ in each sequence, base by base.

I remember getting a call from my student, Stan Hollenberg, while I was at the conference, and he said that Russ had found a homology between the glucocorticoid receptor and the oncogene called $V$-erbA. I immediately left the meeting, drove 90 miles an hour, purchased a new airline ticket to go home, and wrote the first paper showing that the first steroid receptor to be sequenced had homology to an oncogene. This finding linked receptors to oncogenes, suggested that the oncogene was probably a new receptor, and led us to clone the cellular form of the oncogene, which turned out to be the thyroid hormone receptor. That knocked our socks off! We were in another world-completely transformed. That first paper was published in 1985 (Hollenberg SM, Weinberger C, Ong ES, Cerelli G, Oro A, Lebo R, Thompson EB, Rosenfeld MG, Evans RM: The primary structure and expression of a functional human glucocorticoid receptor cDNA. Nature 1985;318:635-641).

\section{How has the study of nuclear receptors evolved since your laboratory cloned the first steroid hormone recep- tor in 1985? For example, how has our fundamental knowledge of the role of these proteins in signal trans- duction changed?}

This has completely changed. It was not understood at all how the hormonal signal was transduced. We now know that there is a single pathway through which all of the nuclear receptors function, and they do this by recruiting either corepressors or coactivators, and that recruitment is controlled by the ligand. We know that these cofactors are enzymes. So the hormonal signal is ultimately transduced into an enzymatic activity at the level of the chromatin template. Much of that activity involves modification of histone tails. That mechanism had not previously even been considered.

\section{How has the understanding of the role these proteins play in disease evolved?}

It was known that these proteins were important, because many hormones were already being used as drugs to treat disease, but we did not know how they worked. With the receptors in hand, we were able to do screening to identify the role of the receptors in the disease and the effects of mutations that contribute to disease. The ability to study the distribution of the receptors in specific tissues-and one of our goals is to determine the distribution of every receptor in every tissue-reconfigures the landscape of how these receptors contribute to disease. Because they are transcriptional regulators, it changes our view of normal physiology as well. In one classic view, organ physiology is a process controlled by receptors and hormones. But, in the modern view, it is a matrix of transcription factors, receptors, cofactors, and the target genes, creating a molecular matrix. That is a very different way of thinking about physiology. From the inside of the matrix you see a complex of transcriptional circuits that for each segment is relatively simple, but their intersection creates a true network. On a molecular basis, this is a complex and codependent series of genetic regulatory events. Our thinking has been completely transformed.

\section{How has the view of the structure and size of the nu- clear receptor family changed?}

We now know that it is a superfamily of a defined number, and that all of the members probably have an interrelated function. The superfamily gives us an entirely new collection of potential therapeutic targets, draws our attention to new physiological pathways, and provides us with the tools to dissect those pathways. We can develop knock-outs and knock-ins and new ligands.

\section{What have been the effects of the evolving assay meth- ods and technologies used to study these receptors?}

The assay methods have become so powerful, yet so easy to employ, that rapid development of new classes of drugs to these receptors is possible. The field has transformed from a crawl to a walk to a run. Now it is a sprint, and it has been one continuous sprint for nearly 20 years.

In the mid-1990s, your laboratory described the potential consequence of nuclear receptor heterodimerization (Forman BM, Umesono K, Chen J, Evans RM: Unique response pathways are established by allosteric interactions among nuclear hormone receptors. Cell 1995; 81:541-550). What is the implication to drug development and particularly assay design of these complex interactions?

With the discovery of the superfamily, these receptors were divided into two groups: the steroid receptor subfamily, and the nonsteroid receptors, which we called the orphan receptors. The existence of the orphan receptors suggested the existence of potential new physiologic circuits and potential new ligands. We were able to find the first ligand for an orphan receptor by setting up receptor-based assays and screening with natural molecules or certain types of synthetic chemicals. We discovered that many of the receptors did not work in the reporter assays on their own. They only functioned in the presence of a second receptor, which was RXR (retinoid X receptor). Those receptors are RXR-dependent heterodimers.

The heterodimer appears to be an ancient motif that evolved before the divergence of vertebrates and invertebrates. Drosophila have an RXR homologue. These orphan receptors typically form heterodimers, whereas the 
steroid receptors form homodimers. Once we understood the heterodimer concept, we were able to devise assays that facilitated the discovery of new ligands. Almost all of those new ligands were for important metabolic pathways, such as the control of cholesterol metabolism and fatty acid and triglyceride metabolism. The heterodimerization concept provided the entrée to the process called "reverse endocrinology," in which you take an unknown receptor and find its ligand. That transformed the field and opened up new areas of physiology. It showed that these receptors are very important regulators of lipid metabolism.

In a recent Science article (Lee CH, Chawla A, Urbiztondo N, Liao D, Boisvert WA, Evans RM: Transcriptional repression of atherogenic inflammation: modulation by PPAPס. Science 2003;302:453-457) you and your colleagues described a novel mechanism for PPAR- $\delta$ and its role in atherogenic inflammation. Please explain how these findings fit in with and further expand our current understanding of PPAR molecular endocrinology.

Over the last five years, our lab has made a major effort to study the most neglected of the PPAR subfamily, which is called PPAR- $\delta$. PPAR- $\alpha$ and PPAR- $\gamma$ were more popular because there were drugs for those two receptors, and those drugs were known to be very powerful for promoting lipid metabolism or insulin sensitization. There is no drug targeting PPAR- $\delta$, so interest in it has been low. We believed that if PPAR- $\alpha$ and PPAR- $\gamma$ were so effective in these metabolic areas, it was likely that PPAR- $\delta$ would do something similar. In fact, that is true, but it does that and more.

The paper shows that PPAR- $\delta$ is an important regulator of inflammation. Inflammation is a common theme in a nuclear receptor pathway; for example, glucocorticoids are antiinflammatory drugs - topical formulations to treat rashes, inhaled versions for asthma, pills to reduce joint pain, and various drugs to treat autoimmune diseases such as lupus and rheumatoid arthritis. Additionally, certain cancers, such as leukemias, are very sensitive to glucocorticoids. The paper shows that in the coronary artery lesion involved in heart disease, which is known to be a site of both inflammation and lipid accumulation, the activation of PPAR- $\delta$ reduces inflammation in the lesion and slows progression of the disease.

We have also found that PPAR- $\delta$ activation in other tissues outside of the heart, particularly fat and muscle, promotes energy burning. The activation of PPAR $\delta$ in peripheral tissue results in resistance to weight gain-very dramatic resistance. We are very excited by this, because it suggests that orally active drugs targeting PPAR- $\delta$ may be both antiinflammatory and protective for diet-associated obesity. We think this will become a very exciting target in the next year.
What do you see as current roadblocks to the development of hormone nuclear receptors and transcription factor-targeted drugs?

In the development of the next generation of drugs, a number of assays could be implemented. Assays for the receptors themselves are subject to patent protection, and these patents could limit certain types of screening approaches, introducing freedom-to-operate issues. The use of transcriptional cofactors could also be dependent on patents. Furthermore, we can patent the use of a receptor to screen compounds and mechanisms of receptor activation or antagonism. One of the challenges to the industry is to figure out how to deal with broad enabling technologies.

\section{What are the major mechanistic factors that distinguish a nuclear receptor from a transcription factor-based modulator or drug, for example, a $G$ protein-coupled receptor or ion channel-based modulator?}

All nuclear receptors function through a common pathway that involves transcriptional control-a genetic network. We know the mechanisms by which that network is controlled by each individual receptor, and we know some of the nodal points that connect these networks to create the larger matrix. GPCRs and ion channels are at the cell surface, and the molecules that activate them act at the cell surface, triggering a second messenger, but they do not need to enter the cell. That is a completely different mechanism. The cell surface receptors are not unified; they function through distinct pathways.

Endocrine modulators are some of the most biologically potent compounds known (e.g., so-called "endocrine disrupters" such as DDT, PCBs, and dioxins contaminating the environment have had profound effects on fish and wildlife development in the parts-per-billion to partsper-thousands range). What are some of the implications of this in the development of drugs that target nuclear receptors, with respect to safety and specificity? Were any of the problems with hormone replacement therapy (HRT) related to a lack of specificity?

Small chemicals and toxins can bind and activate certain nuclear receptors and cause endocrine disruption, affecting development and fertility, as a consequence of blocking normal hormonal pathways. The endocrine system can be sensitive to certain environmental compounds, such as PCBs. In addition, there may be many other environmental compounds that we just don't know about. In fact, people deliberately take herbal compounds for the molecules present in them that alter certain functions in the body. Many of these compounds are able to bind and activate nuclear receptors. It is very possible that either unintentionally through the environment or deliberately by taking certain herbal compounds or prescription drugs that you can alter your hormonal status. We need 
to be careful and consider this possibility when developing new classes of compounds. The problems with HRT are not really related to that lack of specificity.

\section{Would you please explain how your laboratory has em- ployed targeted gene disruption in mice to elucidate the physiological and pathophysiological role of hormone nuclear receptors?}

We create a knock-out mouse for every receptor we identify. One good example would be PPAR- $\gamma$, the target of the widely used TZD drugs, which are insulin sensitizers. We wanted to know the key tissue in which the TZDs act. Because diabetes and insulin resistance are associated with obesity, we thought that adipose tissue would be the target of the drug. We set up a series of experiments to knock out PPAR- $\gamma$ in adipose tissue, muscle, and liver, and to do the reciprocal, increasing the activity of PPAR- $\gamma$ in adipose tissue, muscle, and liver. This is a way to dissect the tissue-specific function of a receptor. We treat the knock-out mice with the drug and determine whether the drug improves insulin sensitivity in those animals when the receptor is missing in a specific tissue.

This is a very powerful strategy. The development of a disease such as insulin resistance or diabetes is a bit like a Rubik's cube, in that individual tissues contribute to the whole process, and each plays a specific role. You cannot assign responsibility for the pathology to a single tissue. Furthermore, the drug appears to act in distinct ways in the fat to lower certain fatty acids, another way in the muscle to promote insulin signaling and the incorporation of glucose, and yet another way in the liver to reduce glucose output.

Another powerful approach involves knocking out a receptor in the mouse and replacing it with the corresponding human receptor. This is a process we have called humanizing the mouse. By doing so, the receptor now has properties that are more like you would expect to see when a human would be treated with the drug. We have incorporated the human xenobiotic receptor, which is the receptor for foreign drugs, into mice, and the mice now respond with a typically human profile.

\section{What are the primary criteria you would consider nec- essary for a nuclear receptor to be considered for de- velopment as a drug target? Can "orphan" nuclear re- ceptors be considered with limited information regarding their ligands?}

In the pharmaceutical industry, for drug target identification, you would have to know that the receptor plays a specific role in the development of a disease or in its treatment. That typically means that a drug already exists for the receptor. For example, the glucocorticoid receptor is a drug target because there are already drugs targeted to it; similarly, the estrogen receptor is also a drug target. Orphan receptors are not considered drug targets; they are considered risk-based targets because there are no drugs for them. Even if you developed a drug to an orphan receptor, you would not know whether it would be able to treat a human disease, would be safe, and would raise enough money to justify its development. So, although these are not popular targets for drug companies, they do represent their future. These risk-based, orphan receptors are being explored in the biotechnology industry. The ones shown to have potential value will be developed in partnerships with pharmaceutical companies, and new drugs will be developed. They will have to fit in with the particular therapeutic areas that a pharmaceutical company has established in its developmental pipeline.

Please provide a brief history of Ligand Pharmaceuticals, your role in the founding of the company, and its current status among biotechnology companies today? How did your academic work lead you to the founding of such companies as Ligand and X-ceptor?

Ligand Pharmaceuticals was founded in 1988, and I am the sole scientific founder of the company. It was designed to exploit the new tools in nuclear receptor biology, to identify new compounds, and to open the area of orphan receptors as bona fide drug targets. Ligand has grown steadily, with five prescription drugs approved and about ten partnerships with pharmaceutical companies that have led to drugs in advanced stages of development. It has been very successful. I would like to see it be even more successful, but it is very difficult to develop drugs and most difficult to develop blockbuster drugs.

If you want to see the commercial potential of basic research, you have to move it into a company. For example, we discovered an orphan ligand called 9-cisretinoic acid-it is the ligand for RXR. But it took a $\$ 100$ million effort to convert that into an FDA-approved drug. No academic laboratory can do that on its own. You would almost certainly doom the drug to failure because of a lack of knowledge of how to proceed, how to design the clinical programs, and how to prove the efficacy and safety of the drug to the FDA.

Ligand acquired all of the rights to the nuclear receptor superfamily from our laboratory. That turned out to be too much for one company. So Ligand spun out a second entity called X-ceptor, which is solely devoted to orphan receptors. That poses a much higher risk, a risk that is being shouldered by investors, by risk-based venture capital. Ligand, on the other hand, is a publicly traded company. Xceptor's goal is to breathe knowledge into orphan receptors in the context of making them drug targets by forming strategic partnerships with companies and helping those companies develop drugs to individual receptors. This is a way to make orphan receptors come into the mainstream.

Thank you, Dr. Evans. 